\title{
THE FERROELECTRIC PLASMA THRUSTER
}

\author{
Mark Kemp \\ Dr. Scott Kovaleski, Dissertation Supervisor
}

\begin{abstract}
Micropropulsion is an enabling technology for microspacecraft. As requirements for microspacecraft missions become more demanding, so do the requirements for micropropulsion. Therefore, existing technologies require improvement, or new technologies need to be developed. The Ferroelectric Plasma Thruster (FEPT) is a new technology for microspacecraft propulsion. This dissertation is a demonstration of this technology and its characteristics.

To produce thrust, the FEPT produces a plasma and a beam of ions. The acceleration of these ions away from the thruster is the mass-transfer mechanism of the thruster. Plasma parameters were measured such as average ion current, ion energy, ion species, and average power dissipated. Photography was performed including open-shutter photography and framing photography. These measured parameters were used to make a calculation of the thrust due to ions.
\end{abstract}

For a more direct measurement of thrust, a micro-thrust stand was designed, built, and characterized. In addition, the mass flow rate from the thruster was measured by weighing the FEPT before and after operation. Combining these two measurements leads to a calculation of the specific impulse. Measured parameters include a thrust of $\sim 70 \mu \mathrm{N}$, specific impulse of $\sim 400$ $\mathrm{s}$, average power of $\sim 6 \mathrm{~W}$, and impulse bits of $<1 \mathrm{nN} * \mathrm{~s}$.

Advantages of the FEPT include its small mass of less than $6 \mathrm{~g}$ and its inherent simplicity. Only a single, low-power rf power supply is needed to form plasma and accelerate particles. Also, electrons are also expelled from the thruster which potentially removes the necessity of a separate neutralizer. Finally, the impulse bits delivered by the FEPT are extremely small which leads to very fine control of microspacecraft attitude. 